\title{
On the Maximum Modulus of a Polynomial
}

\author{
V.K. Jain
}

ABSTRACT: For a polynomial $p(z)$ of degree $n$, having no zeros in $|z|<1$ Ankeny and Rivlin had shown that for $R \geq 1$

$$
\max _{|z|=R}|p(z)| \leq \frac{R^{n}+1}{2} \max _{|z|=1}|p(z)| .
$$

Using Govil, Rahman and Schmeisser's refinement of the generalization of Schwarz's lemma we have obtained a refinement of Ankeny and Rivlin's result. Our refinement is also a refinement of Dewan and Pukhta's refinement of Ankeny and Rivlin's result. 